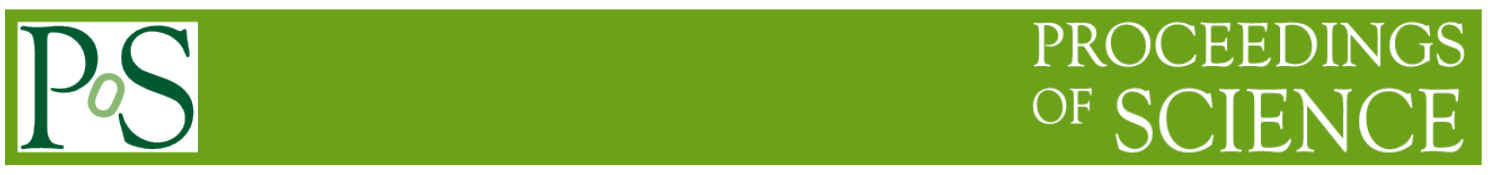

\title{
The Moving Objects Tracking Algorithm in Soccer Robot System Based on Extended Kalman Filter
}

\author{
Tianwei $\mathbf{N i}^{12}$ \\ Department of Electronics and Information Engineering \\ Hohai University Wentian College, Maanshan 243031 Anhui China \\ E-mail: tianwei56890126. com

\section{Jinzhu Lin} \\ Department of Electronics and Information Engineering \\ Hohai University Wentian College, Maanshan 243031 Anhui China \\ E-mail: jinzhu_linesina.com
}

In the RoboCup 3D simulation system, a number of important information in the world model, such as the agent coordinate and the soccer ball coordinate information cannot be acquired directly from the analytical sensor information but through necessary calculations. As the traditional Kalman filter algorithm has some limitations in locating and tracking soccer, this paper puts forward the moving objects tracking algorithm in the soccer robot system based on Extended Kalman Filter (EKF). In this project, the moving object considers the soccer ball as an example. The simulation results show that the algorithm can improve the shoot precision of the robot soccer ball and the accuracy of the goalkeeper robot to save the ball in some degree.

CENet2017

22-23 July 2017

Shanghai, China

\footnotetext{
${ }^{1}$ Speaker

${ }^{2}$ This work was supported by the Major Teaching Reform Research Project of Anhui Province(NO.:2016jyxm0907) and Scientific Research Project of Hohai University Wentian College(NO.:WT15001)
} 


\section{Introduction}

The RoboCup3D robot soccer simulation is a project of RoboCup China Open and China robot competition every year. In the whole system of RoboCup3D robot program, it needs to use a virtual soccer field to describe the world. There are static objects in the soccer field, such as the field information and the mark pole coordinates, etc. and dynamic objects, such as the agent perception and the agent action, etc.. The world model stores and explains the current global information for the soccer robot to read. It is the agent through a variety of sensors to get the perceptual information to save in the field so as to make decisions for the players and speculate on the optimal action under a given situation. The world model can be divided into four layers: the basic information storage layer, the update layer, the state prediction layer and the high level decision layer. See Fig. 1 for the structure of the world model.

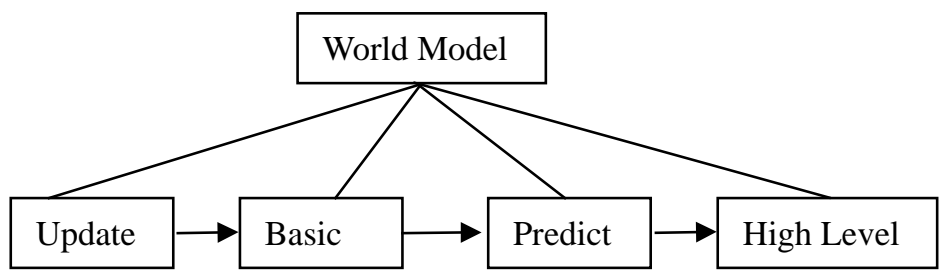

Figure 1: Structure of Four-layer Model

In the world model, the update layer mainly carries on analysis to the information which transmits from the Server, and then updates the information in the Basic layer, stores the calculated data to the corresponding data structure. There are the least squares method [1], Kalman filter algorithm [2] and BP neural network algorithm [3], etc. which are commonly used in the update layer of the world model. But the model also features some shortcomings and defects in its application. The least squares method depends on the visual information to a great extent. If there's any error, to any extent, in the position information acquired by the visual systems, larger degrees of deviation will exist in the results as previously predicted [4]. The Kalman filter algorithm is an efficient self-regression filter, which can estimate the state of the dynamic system from a series of incomplete and noisy measurements, but its disadvantage lies in the slow signal convergence rate [5]. Therefore, in view of the complex motion characteristics of the target, when the velocity of the motion is changed, the use of a single positioning algorithm cannot achieve fast and accurate tracking. The BP neural network algorithm depends on specific environmental conditions to a great extent, but the algorithm has a certain degree of space and time complexity. Considering the inherent defects of the above algorithm, this paper presents a moving objects tracking algorithm in soccer robot system based on the extended Kalman filter. The algorithm features low complexity and easiness of achievement; besides, it can accurately predict the location of the soccer ball in the soccer robot system. At the same time, the algorithm has been successfully applied to the simulation robot competition. 


\section{Analysis of Traditional Kalman Filtering Algorithm}

The basic Kalman filter (KF) algorithm was proposed by Kalman (R. E. Kalman) in early 1960s. The dynamic information is predicted to achieve the optimal estimation of the system's state by the KF algorithm based on the input and output data of the system [6][7].

The implementation of Kalman filter is closely related to the filtering equation by comparing the estimated value of the previous time state with the value actually measures at the current time. The updated estimation of the current state variable is implemented.Thus the optimal estimation of the current state can be obtained. According to the state variable $\boldsymbol{x} \in \boldsymbol{R}^{n}$, for a linear discrete system, the Kalman filter may use Formula (2.1) to represent the state equation of the system.

$$
X_{k}=A \bullet X_{k-1}+B \bullet U_{k}+W_{k}
$$

If the observed variable is $z \in R^{n}$, the observation equation is shown as follows:

$$
Z_{k}=H \bullet X_{k}+V_{k}
$$

Where ${ }^{X_{k}}$ Is the state vector of the system at the time of K, A and B are constant of the system, $U_{k}$ is the control vector of the system at the time of $\mathrm{K}, W_{k}$ is the system process noise sequence, $Z_{k}$ is the observation value at the time of $\mathrm{K}, \mathrm{H}$ is the observation matrix of the system, $V_{k}$ is the observation noise sequence.

Assuming that the K observation and the optimal estimate of the system were $X_{k}$ and $\hat{X}_{k}$ respectively, the linear combination $A \bullet \hat{X}_{k-1}+B \bullet Z_{k}$ is used as the filtering estimate of $x_{k}$. The formula is as follows:

$$
\hat{X}_{k}=A \bullet \hat{X}_{k-1}+B \bullet Z_{k}
$$

The formula of systematical error and mean square error are shown as follows:

$$
\begin{gathered}
E_{k}=X_{k}-\hat{X}_{k} \\
P_{k}=E\left[E_{k} \bullet E_{k}{ }^{T}\right]=E\left[\left(X_{k}-\hat{X}_{k}\right) \bullet\left(X_{k}-\hat{X}_{k}\right)^{T}\right]
\end{gathered}
$$

By combining Formula (1.1) and Formula (1.2), the Kalman filter time updating equation is obtained:

$$
\begin{gathered}
\hat{X}_{k}^{-}=A \bullet \hat{X}_{k-1}+B \bullet U_{k-1} \\
\hat{P}_{k}^{-}=A \bullet P_{k-1} \bullet A^{T}+Q
\end{gathered}
$$

Where $\hat{X}_{k}^{-}$represents a priori estimation of $X_{k}$ and $\hat{P}_{k}^{-}$represents a priori estimate of $P_{k}$

The yield value formula of Kalman filter is as follows:

$$
K_{k}=P_{k}^{-} \bullet H^{T} \bullet\left(H \bullet P_{k}^{-} \bullet H^{T}+R\right)^{-1}
$$

The mean square error for update is as follows: 


$$
P_{k}=\left(I-K_{k} \bullet H\right) \bullet P_{k}^{-}
$$

Based on the Kalman filter time update equation and the state equation to estimate the current time or the state value after current moment, the robot makes further relatively accurate action.

\section{Extended Kalman Filter Location and Tracking Algorithm}

Extended Kalman Filter (EKF) algorithm is developed on the basis of the traditional KF algorithm. With the diversity of the target motion characteristics, the observation environment becomes very complex in the target tracking system. The least square localization algorithm is affected by noise of the measured value. The traditional Kalman filter can get the dynamic target information and take the corresponding mechanism to eliminate the negative effect caused by the noise, and finally get the optimal estimation of the target location in a system. The performance comparison of the two algorithms is shown in Table 1.

\begin{tabular}{c|c|c}
\hline Location algorithms & RMSE/cm & Tracking reaction time/s \\
\hline $\mathrm{KF}$ algorithm & 2.57 & 4.336 \\
\hline Least method squares algorithm & 6.41 & 0.5 \\
\hline
\end{tabular}

Table 1: Performance Comparison of the Two Traditional Algorithms

Unfortunately, due to the speed of the target mutation, the KF algorithm has a certain degree of delay problem, making it difficult for the network to track the target precisely and sometimes even loss of the target. But the KF can be used to estimate the dynamic object location and prediction in the RoboCup3D simulation game. The EKF algorithm applied to the discrete time nonlinear dynamic systems is often used in the target tracking system. The basic idea of EKF is the linear system of nonlinear system, and then to apply Kalman filter. In this sense, EKF is a sub-optimal filter.

Considering the nonlinear system, the state equation and the measurement equation are shown as follows respectively:

$$
\begin{aligned}
x_{k+1}=f\left(x_{k}, u_{k}\right)=\varphi x_{k}+\Gamma u \\
\quad z_{k}=h\left(x_{k}, v_{k}\right)=h\left(x_{k}\right)+v
\end{aligned}
$$

The state variables is assumed to be linear functions, while the observed variables is nonlinear functions. At the same time, the state estimation value of ' $\mathrm{k}-1$ ' moment is $\hat{x}_{k-1 / k-1}$, the estimation error covariance matrix is $P_{k-1 / k-1}$, and the extended Kalman filter recursive method is as follows [8]:

Step 1: according to the state equation, the target state is further extrapolated to obtain $\hat{x}_{k / k-1}$.

Step 2: we can acquire a Taylor expansion of the nonlinear observation equation $h(x)$ at $\hat{x}_{k / k-1}$ and take the first item. 


$$
\begin{gathered}
h\left(x_{k / k}\right) \approx h\left(\hat{x}_{k / k-1}\right)+\left.\frac{\partial h}{\partial x^{T}}\right|_{x=\hat{x}_{k / k-1}}\left(x-\hat{x}_{k / k-1}\right) \\
=h\left(\hat{x}_{k / k-1}\right)+H\left(\hat{x}_{k / k-1}\right)\left(x-\hat{x}_{k / k-1}\right)
\end{gathered}
$$

Step 3: we are going to plug Formula (3.3) into Formula (3.2) to get approximate linear model:

$$
z=H\left(\hat{x}_{k / k-1}\right) x+h\left(\hat{x}_{k / k-1}\right)-H\left(\hat{x}_{k / k-1}\right) \hat{x}_{k / k-1}+v
$$

Step4: the renewal equation of state filter is shown as follows:

$$
K=P_{k / k-1} H^{T}\left(H P_{k / k-1} H^{T}+R\right)^{-1}
$$

The renewal equation of the gain matrix is shown as follows:

$$
\hat{x}_{k / k}=\hat{x}_{k / k-1}+K\left[z-z\left(\hat{x}_{k / k-1}\right)\right]
$$

The renewal equation of the error covariance matrix is shown as follows:

$$
P_{k / k}=(I-K H) P_{k / k-1}(I-K H)^{T}+K R K^{T}
$$

In fact, the operation principle of EKF and KF are similar, predicted and corrected. A time to get the posterior estimations will provide data support for the next time a priori estimation. The agent is very important for the location and speed of the soccer ball because all the players have to pay attention to the position of the soccer ball in the RoboCup3D competition. Based on the idea of the above method, efforts are made to realize the prediction of the soccer ball position. In this paper, we design a moving objects tracking algorithm in soccer robot system based on the extended Kalman filter, which can accurately predict the position of the ball and obtain the effect of the close to the real value.

\section{Motion Model of Soccer Robot in RoboCup3D Simulation}

Firstly, we need to consider the nonlinear motion state of the ball in the simulation platform, the mathematical model of the object, the visual coordinates of the target object to make some changes in the angle of view, such as a certain rotation and translation operations. Secondly, the vision coordinate of the target object is transformed into the coordinate of the fixed coordinate system with respect to the robot's torso in the field. Finally, EKF models will be designed respectively according to both $\mathrm{X}$ and $\mathrm{Y}$ axes of a ball on a spherical surface.

Design the ball state model for soccer ball in robocup3d simulation platform, as shown in Formula (4.1):

$$
\left(\begin{array}{c}
x_{t} \\
x_{t}^{\prime}
\end{array}\right)=\left(\begin{array}{c}
x_{t-1}+x_{t-1}^{\prime} \Delta t \\
x_{t-1}^{\prime}
\end{array}\right)+\zeta_{t}
$$

Where $t$ is the sampling time interval, $x$ is the position information, $x^{\prime}$ is the speed information, $\Delta t$ is the length of simulation numerical range, $\zeta_{t}$ is the process noise.

The mathematical model of the soccer ball is as shown in Formula(4.2):

Where $\mathrm{Z}$ is the measurements and $\zeta_{t}$ is the process noise.

$$
Z_{t}=\left(\begin{array}{ll}
0 & 1
\end{array}\right) x_{t}+\zeta_{t}
$$

The prediction model of the soccer ball is as shown in Formula (4.3): 
Where $\Delta x_{t}$ is the translation of the visual coordinate information, $\Delta y_{t}$ is the rotation of the visual coordinate information. By using EKF algorithm to locate the target object, it will always focus on the change of the state of the object in the moving field and not predict the value of the object in the vertical direction.

$$
\left(\begin{array}{c}
x_{t} \\
y_{t} \\
0 \\
0
\end{array}\right)=\left(\begin{array}{cccc}
\cos \left(-\Delta \theta_{t}\right) & -\sin \left(-\Delta \theta_{t}\right) & 0 & -\Delta x_{t} \\
\sin \left(-\Delta \theta_{t}\right) & \cos \left(-\Delta \theta_{t}\right) & 0 & -\Delta y_{t} \\
0 & 0 & 1 & 0 \\
0 & 0 & 0 & 1
\end{array}\right)\left(\begin{array}{c}
x_{t} \\
y_{t} \\
0 \\
0
\end{array}\right)
$$

A single agent will receive a visual information within the maximum simulation period (three simulation cycles), and this is processed in a timely manner. In this paper, the simulation period of EKF algorithm is also provided for three simulation cycles. In this sense, when the agent doesn't see the target object (refers to the ball herein) in the specified time of more than $3000 \mathrm{~ms}$, you need to resolve other agent information in exchange for the ball state.

\section{Experimental Verification and Analysis}

The estimation result of the moving object trajectory based on EKF is given by MatLab simulation, as shown in Fig. 2. The EKF algorithm is used for filtering observations obtained by update, which is close to the real value effect so that it makes the location information in the simulation system is more concentrated, either forwards or the goalkeeper can make a success of making a save.

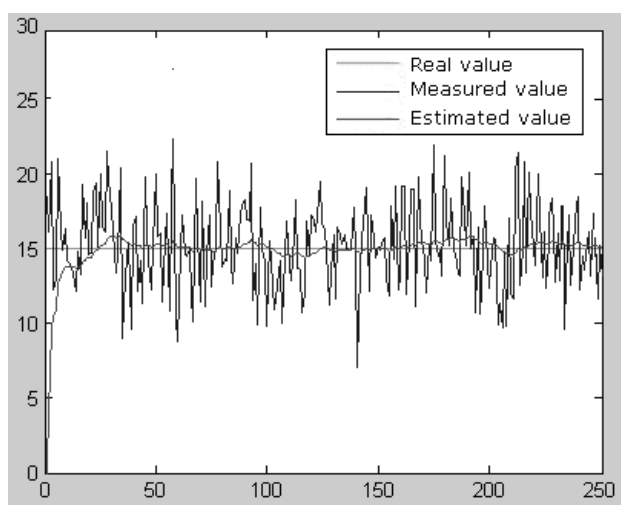

Figure 2: Simulation Results of EKF



Figure 3: Performance Comparison of the Speed

Algorithm

Mutation Case

In case of the speed mutation of the moving target, the EKF algorithm and the KF algorithm are simulated respectively. Assuming that the target moves at the speed of $0.6 \mathrm{rad} / \mathrm{s}$ to the target and stops suddenly in this experiment, in this paper, the root of the equation is solved by iteration method and calculated by five decimal places. The accuracy of the calculation results for $E=10^{4}$ and the step $h=0.1$ s, the iterative results are shown in Fig. 3 .

Compared with the standard KF algorithm, when the target speed doesn't radically change, the number of iterations has no great difference. When the speed of moving target suddenly reduces to 0 right now, the iteration number of EKF is smaller than KF; furthermore, the convergence rate of EKF is much faster, the EKF track is more smooth and accurate, and the tracking effect is better. 


\section{Conclusion}

In this paper, a new moving objects tracking algorithm is proposed for soccer ball locating and tracking based on the extended Kalman filter. This paper introduces the idea of EKF algorithm for soccer ball positioning in detail and designs the model of the ball, the prediction model of the ball etc. in the robocup $3 \mathrm{~d}$ simulation platform system. The simulation results show that the moving object tracking algorithm is efficient and feasible in the soccer robot system so that it can be used to improve the prediction accuracy of the soccer ball position. In this sense, it has laid foundation for kicking the ball and succeeding in making a save. Then, we will use the moving objects tracking algorithm in the robocup3d simulation game to accurately locate the position of the robot and soccer ball, improve the accuracy of soccer robot shooting and making a save.

\section{References}

[1]JULIER S J,UHLMANN J K,DURRANT-WHYTE H F.A New Approach for Filtering Nonlinear Systems $[\mathrm{C}] / /$ Proceedings of the American Control Conference. Seattle, Washington: [s.n.],1995:1628-1632.

[2]KALMAN R E.A new approach to linear filtering and prediction problems[J].Trans ASME J Basic Engineering, 1960,32:35-44.

[3]DAUM F E.Nonlinear filters:beyond the Kalman filter[J].IEEE AES Systems Magazine,2005,20(8):57-69.

[4]ZHAO Xiao, WANG Ming, LI Xiao-ming. Application of extended Kalman filter algorithm in intelligent robot soccer competition[J].Journal of Mechanical \& Electrical Engineering,2012,29(3):334-338.

[5]Yang Liu, Sun Zhen-dong. EKF-based adaptive sensor scheduling for target tracking[C]//IEEE International Symposium on Information Science and Engieering,2008:171-174.

[6]ZHU JI-Hua, ZHENG Nan-ning, YUAN Ze-jian. A SLAM algorithm based on the central difference Kalman filter[C]//Intelligent Vehicles Symposium IEEE. Xian:[s.n.],2009:123-128.

[7]Greg Welch, Gary Bishop. An introduction to the Kalman filter[J]. University of North Carolina at Chapel Hill, 1995(7):127-132.

[8]ZHANG Rui SHI Linan. Tracking of Maneuvering Target Based on Extended Kalman Filter[J]. Aerospace Control,2012,30(3):12-18. 